To be submitted to The Astrophysical Journal

\title{
Merging White Dwarf/Black Hole Binaries and Gamma-Ray Bursts
}

\author{
Chris L. Fryer, S. E. Woosley \\ Lick Observatory, University of California Observatories, \\ Santa Cruz, CA 95064 \\ cfryer@ucolick.org \\ Marc Herant \\ Washington University School of Medicine, \\ Box 8107, 660 S. Euclid \\ St. Louis, MO 63110 \\ Melvyn B. Davies \\ Cambridge Institute for Astronomy, \\ Madingley Road, Cambridge CH30HA
}

\begin{abstract}
The merger of compact binaries, especially black holes and neutron stars, is frequently invoked to explain gamma-ray bursts (GRB's). In this paper, we present three dimensional hydrodynamical simulations of the relatively neglected mergers of white dwarfs and black holes. During the merger, the white dwarf is tidally disrupted and sheared into an accretion disk. Nuclear reactions are followed and the energy release is negligible. Peak accretion rates are $\sim 0.05$ $\mathrm{M}_{\odot} \mathrm{s}^{-1}$ (less for lower mass white dwarfs) lasting for approximately a minute. Many of the disk parameters can be explained by a simple analytic model which we derive and compare to our simulations. This model can be used to predict accretion rates for white dwarf and black hole (or neutron star) masses which are not simulated in this paper. Although the mergers studied here create disks with larger radii, and longer accretion times than those from the merger of double neutron stars, a larger fraction of the merging star's mass becomes part of the disk. Thus the merger of a white dwarf and a black hole could produce a long duration GRB. The even rate of these mergers may be as high as $10^{-4} \mathrm{yr}^{-1}$ per galaxy.
\end{abstract}


Subject headings: Gamma-Rays: Bursts, Black Hole Physics, Accretion: Accretion Disks, Stars: White Dwarfs

\section{Introduction}

As evidence supporting the extra-galactic nature of gamma-ray bursts (GRB's) mounts (Metzger et al. 1997; Frail et al. 1997), the class of GRB models based on hyper-accreting black holes is becoming the favorite mechanism for driving these explosions (e.g., Popham, Woosley, \& Fryer 1998; Eberl, Ruffert, \& Janka 1998). Calculations show that a fraction of the gravitational potential energy released as the material in the disk $\left(M_{\text {disk }} \approx 0.1-5 \mathrm{M}_{\odot}\right)$ accretes into a small black hole $\left(M_{\mathrm{BH}} \approx 3-10 \mathrm{M}_{\odot}\right)$ can be converted into a "fireball" which produces the observed gamma-rays (Meszaros \& Rees 1992). Such systems form in collapsars or hypernovae (Woosley 1993, 1996; Paczynski 1997) and in the merger of compact binaries consisting of: two neutron stars or a neutron star and a black hole (Paczyński 1991; Narayan, Paczyński, \& Piran 1992); a helium star and a black hole (Fryer \& Woosley 1998), and, the topic of this paper, a white dwarf and a black hole.

Several mechanisms have been proposed to facilitate the conversion of potential energy into GRB explosion energy. Due to the high densities involved during the merging process, the potential energy may be emitted in the form of neutrinos, and the subsequent annihilation of these neutrinos could power a GRB (Goodman, Dar, \& Nussinov 1987). Meszaros \& Rees (1992) first pointed out the advantages of disk geometry for enhancing neutrino annihilation. However, unless the accretion rate is very high, over a few hundredths of a solar mass per second, Popham et al. (1998) have shown that neutrino emission is inefficent. The energy released in the disk is advected into the hole. Alternatively, and especially for lower accretion rates and lower disk viscosity, the stretching of magnetic field lines and reconnection or rotationaly generated electric potentials can convert the black hole rotation energy (or the potential energy of the accreting material) into a relativistic jet and drive a GRB (Blandford \& Znajek 1977; MacDonald et al. 1986; Paczynski 1991,1997; Woosley 1996; Meszaros \& Rees 1997, Katz 1997). In each of these models, the formation of an accretion disk is an essential ingredient.

One scenario to form binaries consisting of a black hole and a white dwarf (WD/BH binaries) begins with main-sequence stellar systems having extreme mass ratios $\left(M_{\text {primary }}>25-35 \mathrm{M}_{\odot}, M_{\text {secondary }} \approx 1-10 \mathrm{M}_{\odot}\right)$. The formation scenario for white dwarf binaries with neutron star companions is similar, only with a primary star mass between 
10 and $25-35 \mathrm{M}_{\odot}$. As the massive star evolves off the main sequence, a common envelope phase may occur that ejects the primary's hydrogen envelope. Beyond some critical mass (roughly $25-35 \mathrm{M}_{\odot}$ ), massive stars are thought to form black holes, either by a failed explosion, or through significant fallback (Woosley \& Weaver 1995, Fryer 1998). The more massive primary eventually collapses into a $3-15 \mathrm{M}_{\odot}$ black hole and forms a binary consisting of a black hole and a main-sequence star. As the secondary expands and the orbit shrinks, a second mass transfer phase commences. This phase is observed in the closest of these systems as a low-mass X-ray binary (e.g. J0422+32-Nova Per, 2023+338 Nova Cyg). Roughly 20 binary systems with a black hole and a low-mass companion have been observed and many, as yet undetected systems may exist (see Tanaka \& Shibazaki, 1996, for a review). These systems generally involve main-sequence secondaries which lose a significant amount of their mass while still burning hydrogen and will not evolve into massive $\left(>0.5 \mathrm{M}_{\odot}\right)$ white dwarfs. However, slightly wider binaries will not undergo mass transfer until after the secondary has evolved off the main sequence and will produce the systems which we will model in this paper. Unfortunately, the formation rate of the observed low mass X-ray binaries is difficult to determine, and estimating the number wider systems from observations is impossible.

An alternative evolution scenario for WD/BH binaries begins with less extreme mass ratios in a system where the primary initially forms a neutron star in a supernova explosion. As the secondary expands off the main-sequence and a common envelope phase ensues, the neutron star accretes rapidly via neutrino emission and eventually collapses to a low mass black hole (see Fryer, Benz, \& Herant 1996, Bethe \& Brown 1998). Because this common envelope phase happens so quickly, it is unlikely that systems evolving through this scenario will be observed, and no merger rate can be predicted from the observations.

Unfortunately, uncertainties in black hole formation and binary evolution also make it difficult to make any firm predictions using population synthesis studies, but the merger rate is likely to lie in the range from $10^{-7}-10^{-4} \mathrm{yr}^{-1}$ per Milky-Way like galaxy (Fryer, Woosley, \& Hartmann 1998). The large uncertainties in the merger rate are primarily due to uncertainties in the critical mass beyond which massive progenitor stars collapse to black holes and in the kick imparted to black holes. Although a range of white dwarf masses can form in binaries, Fryer et al. find that most of the close systems which eventually merge consist of black holes with massive white dwarf companions $\left(M_{\mathrm{WD}} \gtrsim 0.9 \mathrm{M}_{\odot}\right)$.

Black holes and white dwarfs can also merge through collisions in dense star regions such as in galactic centers and globular clusters. Sigurdsson \& Rees (1997) predict a neutron star/white dwarf merger rate of $\sim 10^{-7} \mathrm{yr}^{-1}$ per galaxy. Low mass black holes will merge with white dwarfs roughly at the same rate (or an order of magnitude less) 
depending upon the black hole formation rate. This rate is comparable to the merger rate predicted by Quinlan \& Shapiro (1987, 1989, 1990). Depending on the beaming fraction, this rate is certainly sufficient to give the observed GRB statistics (Wijers et al. 1998), provided, of course, that the merger produces a GRB.

In this paper, we model the merger of black holes and white dwarfs on the computer using a three-dimensional hydrodynamics code based on the Smooth Particle Hydrodynamics (SPH) method (Benz 1990). We follow the merger from the initial Roche-lobe overflow through the complete disruption of the white dwarf into a disk. Roche-lobe overflow for compact objects differs from that for giant stars in several ways: (a) the closer separations involved in the Roche-lobe overflow of compact objects allows gravitational radiation to play an active role in the orbital evolution, (b) due to the degeneracy of the compact object, its radius increases as it loses mass, and (c) the orbital angular momentum is far from conserved (三 the mass transfer is not "conservative"). We discuss this physics, applicable to most compact object mergers, in $\S 2$. A description of the code along with a presentation of the simulations, including a comparison to the analytic solution of $\S 2$, is given in $\S 3$. We conclude with a discussion of the accretion disks formed in these mergers and their suitability as GRB models.

\section{Accretion Disk Formation}

Whether or not the accretion disks formed in WD/BH mergers produce the necessary GRB explosion energies is determined by the accretion rate and the angular momentum of that disk, which in turn, depends upon the size and mass of the accretion disk. It is important to know, then, how quickly, and at what radius, the white dwarf is torn up by the gravitational potential of the black hole and transformed into an accretion disk which might fuel a GRB. One might naively assume that, since the white dwarf is less massive than the black hole $\left(M_{\mathrm{BH}} \sim 3-10 \mathrm{M}_{\odot}, M_{\mathrm{WD}} \sim 0.5-1.3 \mathrm{M}_{\odot}\right)$, that stable accretion will occur and the white dwarf will slowly accrete onto the black hole over many orbital periods. However, as we shall discuss in this section, several aspects of physics conspire to destabilize this mass transfer, leading to the rapid transformation of most of the white dwarf into an accretion disk. 


\subsection{Gravitational Radiation}

Gravitational radiation plays an important role in the merging of double neutron star or black hole/neutron star systems. For these systems, the emission of gravitational waves tightens the binaries on timescales comparable to those of the hydrodynamical evolution. However, white dwarfs fill their Roche lobes at much wider separations where the gravitational wave merging timescale is 1-100yr (depending upon the white dwarf and black hole masses). Although gravitational radiation does cause the orbit to tighten sufficiently to drive the white dwarf to fill its Roche lobe in the first place, once Roche-lobe overflow occurs, the mass transfer rate from the white dwarf onto a disk around the black hole is determined by the transfer of angular momentum and the white dwarf mass-radius relationship which drive unstable mass transfer on much shorter timescales ( minutes).

\section{2. $\quad$ Effects of Degeneracy}

One such destabilizing effect is the inverse relationship of the radii of degenerate objects (neutron star, white dwarf) with respect to mass. For a $\Gamma=5 / 3$ polytrope approximation of a white dwarf equation of state, this relationship is well known (Shapiro \& Teukolsky 1983):

$$
R_{\mathrm{WD}}=10^{4}\left(\frac{M_{\mathrm{WD}}}{0.7 \mathrm{M}_{\odot}}\right)^{-1 / 3}\left(\frac{\mu_{e}}{2}\right)^{-5 / 3} \mathrm{~km}
$$

where $R_{\mathrm{WD}}, M_{\mathrm{WD}}$, and $\mu_{e}$ are the radius, mass, and mean molecular weight per electron of the white dwarf. Our simulated white dwarfs differ slightly from this simple relation due to deviations away from a simple $\Gamma=5 / 3$ polytrope (Fig. 1). Figure 1 also shows the separations at which the white dwarf overfills its Roche lobe. Stable mass transfer would require that as the white dwarf loses mass, its orbit widens to place it just at this critical Roche lobe separation. If the white dwarf binary instead remains at a constant orbital separation, the accretion will quickly become unstable as the white dwarf itself expands. This effect is important for all merging systems involving a compact secondary.

\subsection{Non-Conservative Mass Transfer}

The orbital separation need not remain constant. In conservative mass transfer, when an object accretes onto a more massive companion, orbital angular momentum conservation requires that the orbit expands. However, some fraction of the material can be lost from the system and carry away angular momentum. Hence, although the total angular momentum 
is conserved, the orbital angular momentum of the binary system decreases, and the orbital separation may actually decrease during mass transfer. This "non-conservative" mass-transfer can be parameterized and solved (see Podsiadlowski, Joss, \& Hsu 1992 and references therein). In addition, for mergers with black holes or neutron stars, some of the orbital angular momentum is converted to angular momentum of the accretion disk or to spin angular momentum of the black hole. The change of orbital angular momentum $\left(\delta J_{\text {orbit }}\right)$ of the binary is then given by:

$$
\delta J_{\text {orbit }}=\left[j_{\text {ejecta }}(1-\beta)+j_{\text {disk }} \beta\right] \delta M_{\mathrm{WD}} \frac{2 \pi A^{2}}{P}
$$

where $\beta$ is the fraction of mass lost by the white dwarf that is accreted by the black hole (or becomes part of the black hole's accretion disk), $j_{\text {ejecta }}$ and $j_{\text {disk }}$ are the specific angular momenta (in units of the white dwarf specific angular momentum) of the ejected material and the material which is either accreted onto the black hole or becomes part of the accretion disk. $A$ and $P$ are the orbital separation and period of the binary system. Following the procedure of Podsiadlowski, Joss, \& Hsu (1992), we derive the orbital separation $(A)$ of the binary during mass transfer including the loss of angular momentum to the accretion disk:

$$
\frac{A}{A^{0}}=\frac{M_{\mathrm{WD}}+M_{\mathrm{BH}+\text { disk }}}{M_{\mathrm{WD}}^{0}+M_{\mathrm{BH}}^{0}}\left(\frac{M_{\mathrm{WD}}}{M_{\mathrm{WD}}^{0}}\right)^{C_{1}}\left(\frac{M_{\mathrm{BH}}}{M_{\mathrm{BH}}^{0}}\right)^{C_{2}}
$$

where the values of the constants differ only slightly from those derived by Podsiadlowski, Joss, \& Hsu (1992):

$$
\begin{gathered}
C_{1} \equiv 2 j_{\text {ejecta }}(1-\beta)-2+2 j_{\text {disk }} \beta \\
C_{2} \equiv \frac{-2 j_{\text {ejecta }}}{\beta}(1-\beta)-2-2 j_{\text {disk }}
\end{gathered}
$$

and

$$
M_{\mathrm{BH}+\mathrm{disk}}=\beta\left(M_{\mathrm{WD}}^{0}-M_{\mathrm{WD}}\right)+M_{\mathrm{BH}}^{0},
$$

and where superscript 0 denotes pre-mass transfer phase values.

In Roche-lobe overflow onto compact objects (neutron stars or black holes), much of the angular momentum is placed into a disk around that compact object. For the merger of binaries consisting of a black hole and a neutron star, roughly half of the orbital angular momentum is fed directly into spinning up the black hole (Eberl, Ruffert, \& Janka 1998). For wider Roche-lobe overflow systems (e.g. white dwarf mergers) much of the orbital angular momentum is converted into disk angular momentum and is ultimately ejected through viscous momentum transport in the disk (see Papaloizou \& Lin 1995 for a review). Figure 2 shows the orbital evolution for 0.7 and $1.1 \mathrm{M}_{\odot}$ white dwarfs merging 
with a $3 \mathrm{M}_{\odot}$ black hole for a range of values of $j_{\text {disk }}$ (in terms of the white dwarf specific angular momentum $\left.\equiv j_{\mathrm{WD}}\right)$ and assuming no mass is ejected from the system $(\beta=1)$. The critical separation for Roche lobe overflow, shown in Figure 2, marks the dividing line between stable and unstable mass accretion. If the orbit widens faster than the white dwarf expands, the accretion rate onto the black hole is limited to the gravitational wave timescale $(1-100 \mathrm{yr})$ and the merger will occur on these timescales. However, if $j_{\text {disk }}>0.1,0.3 j_{\mathrm{WD}}$ for $0.7,1.1 \mathrm{M}_{\odot}$ white dwarfs respectively, the mass transfer is unstable. If the accreting material transports all of its angular momentum to the accretion disk, then the angular momentum of the disk is roughly the angular momentum of the material of the Lagrange point. Assuming tidal locking, for the binary systems we model, this angular momentum is roughly: $j_{\text {disk }} \approx\left(A-R_{\mathrm{WD}}\right) / A \sim 0.7 j_{\mathrm{WD}}$ where $A$ is the orbital separation and $R_{\mathrm{WD}}$ is the white dwarf radius. For these high values of $j_{\text {disk }}$, unstable mass transfer is inevitable, and we expect the white dwarf to be tidally disrupted quite rapidly. With an understanding of these physical processes, we are now prepared to examine the hydrodynamical simulations.

\section{Simulations}

For our simulations, we use a three dimensional SPH code (Davies, Benz, \& Hills 1991) with 6000-16000 particles. We employ the equation of state developed by Lattimer \& Swesty (1991) for densities above $10^{11} \mathrm{~g} \mathrm{~cm}^{-3}$ and, for low densities, the equation of state by Blinnikov, Dunina-Barkovskaya \& Nadyozhin (1996). We include a nuclear burning network for temperatures above $4 \times 10^{8} \mathrm{~K}$ (Woosley 1986), though we find that burning is not important, except well within the accretion disk. As we are more concerned with the tidal disruption of the white dwarf, not the accretion of matter in the disk formed from this disruption, we model the black hole (or neutron star) as a point mass and remove particles

which fall within $2-3 \times 10^{8} \mathrm{~cm}$ of the black hole, well before general relativistic effects are important. Similarly, since we are not following the evolution of the accretion disk, the artificial viscosity is not important for our simulations. However, as a check, we have varied the artificial viscosity by an order of magnitude and find it does not effect the radius at which the white dwarf is disrupted or the initial structure of the accretion disk formed by this disruption. The viscosity does effect the rate at which material is accreted onto the black hole, and hence the pair fireball energy, which we discuss in $\S 4$.

With this code, we model the tidal disruption of 4 binary systems consisting of a white dwarf (with masses of $0.7,1.1 \mathrm{M}_{\odot}$ ) and a black hole (with masses of $3,10 \mathrm{M}_{\odot}$ ) and one system consisting of a $1.1 \mathrm{M}_{\odot}$ white dwarf and a $1.4 \mathrm{M}_{\odot}$ neutron star. We follow the evolution from the initial Roche-lobe overflow through the destruction of the white dwarf 
and the formation of an accretion disk (Figs. 3, 4). Our estimate of the separation at which Roche-lobe overflow occurs is not exact, but, for simulations where the initial separation is $30 \%$ greater, no mass transfer takes place(Fig. 3), showing that our initial separation is within $30 \%$ of the actual Roche-lobe overflow separation. We will come back to this error estimate in our discussion of the accretion disk properties at the end of this section.

Before we discuss the disk properties, let us first validate our physical picture of the tidal disruption process. From $\S 2$, we expect the angular momentum of the disk to be roughly $\sim 0.7 j_{\mathrm{WD}}$. The actual simulated angular momentum of the disk is $\sim 0.6 j_{\mathrm{WD}}$ and then increases to $1.0 j_{\mathrm{wD}}$ ' as the white dwarf is disrupted (Fig. 5). Physically, this means that as the white dwarf transfers mass onto a disk around the black hole, the angular momentum at the Lagrange point is being added to the disk. When the white dwarf is finally disrupted, nearly all of its angular momentum is immediately put into the disk, and the average disk angular momentum equals the initial white dwarf angular momentum. The disk must then extract this angular momentum before this material can accrete onto the black hole (see $\S 4$ ).

Because much of the orbital angular momentum is converted into disk angular momentum, the orbital separation will not expand as one might expect in conservative mass-transfer, and the white dwarf is quickly disrupted by tidal forces. As the white dwarf accretes mass, the orbital separation of the white dwarf/black hole binary is described by equation (3). Using equation (3) and assuming no mass is ejected from the system (very little mass is ejected in our simulations, see Figs. 3, 4), we can plot our simulated data along with the derived separations for a range $j_{\text {disk }}$ values (Fig. 6). The remarkable agreement of the best fit of $j_{\text {disk }}$ using equation (3) and the actual $j_{\text {disk }}$ values from Figure 5 suggests that we have indeed found the relevant physics, and that the orbital separation can be estimated by our simple mass-transfer model.

In these simulations, the mass transfer from the white dwarf becomes increasingly unstable as more of the white dwarf expands beyond its Roche radius and accretes onto a disk around the black hole. Our simulations show that after losing $\sim 0.2 \mathrm{M}_{\odot}$, the transfer rate becomes so great that the white dwarf is literally disrupted. This disruption occurs rapidly (in an orbit time), dumping the remaining white dwarf mass into an accretion disk around the black hole. This critical mass loss is the one parameter not determined by our analytic model. Using our simulations to constrain this parameter, we are able to describe both the angular momentum and the mass growth rate of the disk from the tidal disruption

\footnotetext{
${ }^{1}$ The end specific angular momentum of the disk created for each system is given in Table 1 and is $\sim 10^{17} \mathrm{~cm}^{2} \mathrm{~s}^{-1}$.
} 
of the white dwarf.

The specific angular momentum of the disk is given by

$$
j \approx M_{\mathrm{BH}} M_{\mathrm{WD}}^{\text {disruption }} \sqrt{G A /\left(M_{\mathrm{BH}}+M_{\mathrm{WD}}^{\text {disruption }}\right)^{3}}
$$

where $G$ is the gravitational constant, $M_{\mathrm{BH}}$ is the black hole mass, and $M_{\mathrm{WD}}^{\text {disruption }} \equiv M_{\mathrm{WD}}^{\text {initial }}-0.2 \mathrm{M}_{\odot}$ is the white dwarf mass at the time of disruption taken from our simulations. The orbital separation $(A)$ can be derived from equation (3). The mass transfer rate of the white dwarf onto the disk is roughly

$$
\dot{M}=M_{\mathrm{WD}}^{\text {disruption }} / T_{\text {orbit }}
$$

where $T_{\text {orbit }}$ is the orbital timescale for the binary system after the white dwarf has lost $0.2 \mathrm{M}_{\odot}$. These results are summarized in Table 1 and the mass-transfer rate can be compared to the simulated rates shown in Figure 7 . Note that our derived mass-transfer rates are well within a factor of 2 of the simulated rates. The actual accretion rate onto the black hole can not exceed this mass-transfer rate.

Many of these results rely upon our knowing the exact separation where Roche lobe overflow commences. As we have already mentioned, by increasing the separation by $30 \%$, we find no accretion occurs over many orbits, which suggests that the error in the initial separation is less than $30 \%$. If the errors in the initial orbital separation are less than $30 \%$, our maximum mass-transfer rates are accurate to $\$ 30 \%$ and the disk angular momenta are accurate to $\lesssim 15 \%$. Even changing the initial separation by a factor of 2 only results in a factor of 3 change in the maximum accretion rate and a change in the angular momenta by less than $40 \%$.

\section{Accretion Disk Powered GRB's}

Based upon these results, we can now address the viability of WD/BH mergers as a GRB model. The mass transfer rate of the white dwarf onto the black hole accretion disk should, in a steady state, balance the accretion rate into the black hole. The actual accretion rate is determined by the efficiency at which the angular momentum is removed from the disk (Popham, Woosley,\& Fryer 1998):

$$
\dot{M}_{\mathrm{acc}} \approx 0.37 \alpha M_{\text {disk }} M_{\mathrm{BH}}^{1 / 2} r_{\text {disk }, 9} \mathrm{M}_{\odot} \mathrm{s}^{-1}
$$

where $\alpha$ is the standard accretion disk parameter, $M_{\text {disk }}$ and $M_{\mathrm{BH}}$ are, respectively, the

mass of the disk and the black hole in $\mathrm{M}_{\odot}$, and $r_{\text {disk }, 9}$ is the outer disk radius in $10^{9} \mathrm{~cm}$. 
Figure 8 shows the mass of the disk as a function of radius for our two $M_{\mathrm{BH}}=3 \mathrm{M}_{\odot}$ models, from which, given a value of $\alpha$, we can determine the accretion rate onto the black hole. For values of $\alpha<0.5$, the accretion rate is limited by the disk accretion and not the mass-transfer rate. Using equation (9), we estimate the effective disk viscosity $(\alpha)$ from accretion rate onto the black hole of our hydrodynamical simulations to be $\sim 0.1$. However, since $\alpha$ is unknown, in this section we retain it as a free parameter and use the hydrodynamical models to determine the disk radii, a result which does not depend upon the numerical viscosity.

The energy from neutrino annihilation can be estimated by integrating the following approximate fit to the pair luminosity results of Popham, Woosley, \& Fryer (1998):

$$
\log L_{\nu, \bar{\nu}}\left(\operatorname{erg~s}^{-1}\right) \approx 43.6+4.89 \log \left(\frac{\dot{M}}{0.01 \mathrm{M}_{\odot} \mathrm{s}^{-1}}\right)+3.4 a
$$

where $a \equiv j c / G M_{\mathrm{BH}}$ is the spin parameter. This fit is reasonably accurate for accretion rates between 0.01 and $0.1 \mathrm{M}_{\odot} \mathrm{s}^{-1}$. Table 2 gives the maximum energies for each of our simulations. In the optimistic situation where $\alpha>0.5$ and the disk accretion rate equals the mass-transfer rate from the white dwarf into a black hole accretion disk, the disruption of a white dwarf around a black hole cannot explain the most energetic gamma-ray bursts without requiring that the mechanism produce strongly beamed jets. Indeed, with isotropic energy requirements as high as $3 \times 10^{53} \mathrm{erg}$ (Kulkarni et al. 1998), the beaming must be extremely high (the burst must be constrained to $0.1 \%$ of the sky, that is, the beaming factor > 1000). However, a wide range of GRB energies may exist (e.g. GRB980425 - see Galama et al. 1998), and WD/BH mergers may only constitute a subset of the observations. If $\alpha=0.1$, the accretion rate drops by about a factor of 5 . For the most optimistic mergers of a $1.1 \mathrm{M}_{\odot}$ with a black hole, this lowers the accretion rate on the black hole down to $0.01-0.02 \mathrm{M}_{\odot} \mathrm{s}^{-1}$ and increases the accretion time, causing a net decrease in the total energy produced by neutrino annihilation of roughly 1-2 orders of magnitude. With beaming factors of $\sim 100, \mathrm{WD} / \mathrm{BH}$ mergers could still explain bursts with inferred isotropic energies between $10^{48}-10^{51} \mathrm{erg}$.

Alternatively, the GRB can be powered by the magnetic fields of the disk, which become stretched and amplified as the material accretes. These magnetic fields then extract the rotational energy of the black hole (or potential energy of the disk material) and power the GRB (Blandford \& Znajek 1977; MacDonald et al. 1986; Paczynski 1991, 1997; Woosley 1993; Hartmann \& Woosley 1995; Thompson 1996; Meszaros \& Rees 1997, Katz 1997, Popham et al. 1998):

$$
L_{\mathrm{rot}}=10^{50}\left(\frac{j c}{G M_{\mathrm{BH}}}\right)^{2}\left(\frac{M_{\mathrm{BH}}}{3 \mathrm{M}_{\odot}}\right)^{2}\left(\frac{B}{10^{15} \mathrm{Gauss}}\right)^{2} \mathrm{erg} \mathrm{s}^{-1}
$$


where $j$ is the specific angular momentum of the black hole and $B$ is the magnetic field strength in the disk. Table 2 lists the total energy that an initially non-rotating black hole would emit over its accretion timescale assuming the magnetic field is $10 \%$ of the equipartition energy, or $0.01 \rho v^{2}$. These high magnetic fields are reasonable if the disk viscosity depends upon the magnetic field strength. In this case, the viscosity is intially $\sim 0$ allowing the disk to continue winding the magnetic field until a sufficiently strong equipartion field is generated which increases the viscosity and allows the disk to accrete.

A successful GRB explosion must also avoid excessive baryonic contamination. The disruption of the white dwarf forms a hot thick disk around the black hole (Figure 9), with some of the matter along the angular momentum axis above the black hole (Table 2). The explosion will force its way along this polar region, sweeping up this material (and possibly pushing some aside). Assuming all of the material is swept along with the burst, we can estimate a lower limit of the Lorentz factors (Table 2). Beaming factors of at least 100 are required to achieve the high Lorentz factors needed to power a gamma-ray burst. Even assuming that beaming factors of 100 do occur, low mass white dwarfs do not produce enough energy (or high enough Lorentz factors) to power a gamma-ray burst. Thus, there is some critical white dwarf mass (between $0.7-1.1 \mathrm{M}_{\odot}$ ) below which no visible GRB will form. Because of the strong dependence of the GRB luminosity on the Lorentz factor, the transition from observed gamma-ray burst to non-detectable explosion is sharp. Those explosions that do not achieve the high Lorentz factors will only be observable in our own Galaxy, and, given the low event rate, will not be detected. We reiterate, however, that most of the merging white dwarfs will be massive (Fryer, Woosley, \& Hartmann 1998) and a large fraction of the merging systems may become GRBs.

The merger of a black hole and a massive white dwarf can produce the energies $\left(10^{48}-10^{51} \mathrm{erg}\right)$ and the high Lorentz factors to explain the long duration GRBs if the bursts themselves are highly beamed (beaming factors $>100$ ). Assuming the GRB rate for isotropic bursts is $10^{-7} \mathrm{yr}^{-1}$ per galaxy of roughly the Milky Way's size (Wijers et al. 1998), the merger rate of massive white dwarfs and black holes with beaming factors $>100$ must be $z 10^{-5} \mathrm{yr}^{-1}$ per galaxy, within the uncertainties of the predicted rates (see $\S 1$ ). Whether or not such narrow beaming can occur remains an open question.

The merger of a neutron star and a white dwarf is a different story. At these accretion rates, Popham, Woosley, \& Fryer (1998) found that much of the energy is advected into the black hole. The hard surface of the neutron star acts as a plug, stopping up this accretion. Unless the neutron star's mass quickly exceeds the Chandrasehkar limit, causing it to collapse to a black hole and removing this plug, the accreting material will flow around the neutron star, building up a spherically symmetric atmosphere. Any explosion from the 
surface will be baryon rich with velocities much less than the speed of light (Fryer, Benz, \& Herant 1994). These outbursts will be too dim to observe beyond our Galaxy, and are too rare to observe within our Galaxy.

This research has been supported by NASA (NAG5-2843 and MIT SC A292701), and the NSF (AST-97-31569). We acknowledge many helpful conversations and communications on the subject of gamma-ray bursts with Andrew MacFadyen, Dieter Hartmann, Thomas

Janka, Max Ruffert, Jonathon Katz and we thank Aimee Hungerford for helpful comments and proofreading.

\section{REFERENCES}

Benz, W., 1990, in Numerical Modeling of Nonlinear Stellar Pulsations: Problems and Prospects, ed. J.R. Buchler (Dordrecht: Kluwer), 269

Blandford, R.D., \& Znajek, R.L., 1977, MNRAS, 179, 433

Blinnikov, S.I., Dunina-Barkovskaya, N.V., \& Nadyozhin, D.K., 1996, ApJS, 106, 171

Davies, M.B., Benz, W., \& Hills, J.G., 1991, ApJ, 381, 449

Eberl, T., Ruffert, M., \& Janka, H.-Th. 1998, A\&A, in preparation

Galama, T.J., et al., 1998, submitted to Nature

Frail, D.A., Kulkarni, S.R., Nicastro, S.R., Feroci, M., \& Taylor, G.B, Nature, 389, 261

Fryer, C.L., Benz, W., Herant, M., 1996, ApJ, 460, 801

Fryer, C.L., \& Woosley, S.E., 1998, 502, L9

Fryer, C.L., 1998, proceedings of the 2nd International Workshop on Laboratory Astrophysics with Intense Lasers, to appear in ApJ supplements, ed. Remington

Fryer, C.L., Woosley, S.E., \& Hartmann, D., 1998, in preparation

Goodman, J., Dar, A., \& Nussinov, S., 1987, ApJ, 314, L7

Hartmann, D.H., \& Woosley, S.E., 1995, Advances in Space Research, vol. 15, no. 5, 143

Janka, H.-T., 1991, A\&A, 244, 378 
Katz, J.I., 1997, ApJ, 490, 633

Kulkarni, S.R., Frail, D.A., Wieringa, M.H, Ekers, R.D., Sadler, E.M., Wark, R.M., Higdon, J.L., Phinney, E.S., \& Bloom, J.S. submitted to Nature 1998

Lattimer, J.M., \& Swesty, F.D. 1991, Nuc. Phys. A, 535,331

MacDonald, D.A., Thorne, K.S., Price, R.H., \& Zhang, X.-H., 1986, in "Black Holes, the Membrane Paradigm", Eds. Thorne, K.S., Price, R.H., \& MacDonald, D.A., Yale Univ. Press

Meszaros, P., \& Rees, M. J., ApJ, 1992, 397, 570

Meszaros, P., \& Rees, M. J., 1997, 482, L29

Metzger, M.R., Djorgovski, S.G, Kulkarni, S.R., Steidel, C.C., Adelberger, K.L., Frail, D.A., Costa, E., \& Frontera, F., 1997, Nature, 387, 389

Narayan, R., Paczyński, B., \& Piran, T. 1992, ApJ, 395,L83

Paczynski, B., 1991, AcA, 41, 257

Paczynski, B., 1997, ApJ, 484, L45

Papaloizou, J.C.B., \& Lin, D.N.C., 1995, ARA\&A, 33, 505

Podsiadlowski, P., Joss, P.C., \& Hsu, J.J.L., 1992, ApJ, 391, 246

Popham, B., \& Woosley, S.E., \& Fryer 1998, submitted to ApJ

Quinlan, G.D., \& Shapiro, S.L., 1987, ApJ, 321, 199

Quinlan, G.D., \& Shapiro, S.L., 1989, ApJ, 343, 725

Quinlan, G.D., \& Shapiro, S.L., 1990, ApJ, 356, 483

Rees, M. J., \& Meszaros, P. 1992, MNRAS, 258, 41P

Ruffert, M., Janka, H.-T., Takahashi, K., Schaefer, G., 1997, A\&A, 319, 122

Shapiro, S.L., Teukolsky, S.A., 1983, Black Holes, White Dwarfs and Neutron Stars, Wiley, New York

Sigurdsson, S., \& Rees, M.J., 1997, MNRAS, 284, 318

Tanaka, Y., \& Shibazaki, N., 1996, ARA\&A, 34, 607 
Thompson, C., 1996, in Gamma-Ray Bursts: 3rd Huntsville Symposium, AIP Conf. Proc 384, eds. C. Kouveliotous, M. Briggs, \& G. Fishman, AIP:New York, 709

Wijers, R.A.M.J., Bloom, J., Bagla, J.S., \& Natarajan, P., 1998, MNRAS, 294, L13

Woosley, S.E., 1986 Saas-Fee Lecture Notes, 1986, in Nucleosynthesis and Chemical Evolution, 16th Advanced Course, Swiss Society of Astrophysics and Astronomy, ed. B. Hauck, A. Maeder, and G. Meynet, Geneva Observatory, 1

Woosley, S.E., 1993, ApJ, 405, 273

Woosley, S.E., \& Weaver, T.A., 1995, ApJS, 101, 181

Woosley, S. E. 1996, in Gamma-Ray Bursts: 3rd Huntsville Symposium, AIP Conf. Proc 384, eds. C. Kouveliotous, M. Briggs, \& G. Fishman, AIP:New York, 709 
Table 1. WD/BH Mergers

\begin{tabular}{lccccc}
\hline \hline Disk Parameters & $\begin{array}{c}M_{\mathrm{WD}}=0.7 \\
M_{\mathrm{BH}}=10\end{array}$ & $\begin{array}{c}M_{\mathrm{WD}}=0.7 \\
M_{\mathrm{BH}}=3\end{array}$ & $\begin{array}{c}M_{\mathrm{WD}}=1.1 \\
M_{\mathrm{BH}}=10\end{array}$ & $\begin{array}{c}M_{\mathrm{WD}}=1.1 \\
M_{\mathrm{BH}}=3\end{array}$ & $\begin{array}{c}M_{\mathrm{WD}}=1.1 \\
M_{\mathrm{NS}}=1.4\end{array}$ \\
\hline$A\left(10^{9} \mathrm{~cm}\right)$ & 4.98 & 3.10 & 2.37 & 1.49 & 1.27 \\
$T_{\text {orbit }}(\mathrm{s})$ & 58.4 & 48.1 & 18.9 & 15.4 & 12.1 \\
$j_{\text {disk }}\left(10^{17} \mathrm{~cm}^{2} \mathrm{~s}^{-1}\right)$ & 1.19 & 1.40 & 1.40 & 1.55 & 1.43 \\
$\dot{M}\left(\mathrm{M}_{\odot} \mathrm{s}^{-1}\right)$ & 0.00856 & 0.01 & 0.0477 & 0.0584 & 0.074 \\
$A^{\text {sim }}\left(10^{9} \mathrm{~cm}\right)^{\mathrm{a}}$ & 5.0 & 3.2 & 2.5 & 1.6 & 1.2 \\
$M_{\mathrm{WD}}^{\text {disr }}\left(\mathrm{M}_{\odot}\right)^{\mathrm{b}}$ & 0.5 & 0.5 & 0.8 & 0.8 & 0.8 \\
$\dot{M}_{\text {peak }}^{\text {sim }}\left(\mathrm{M}_{\odot} \mathrm{s}^{-1}\right)^{\mathrm{c}}$ & 0.012 & 0.008 & 0.063 & 0.079 & 0.075 \\
& & & & & \\
\hline
\end{tabular}

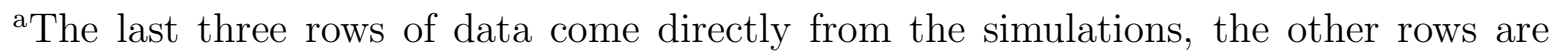
derived from the equations in $\S 2,3$.

${ }^{\mathrm{b}}$ This is the simulated white dwarf mass at the onset of the white dwarf disruption. For our derivations, we assume $M_{\mathrm{WD}}^{\text {disr }}=M_{\mathrm{WD}}-0.2 \mathrm{M}_{\odot}$.

${ }^{\mathrm{c}}$ The peak mass-transfer rate from Figure 5. 
Table 2. Powering a GRB

\begin{tabular}{lcccc}
\hline \hline Observables & $\begin{array}{c}M_{\mathrm{WD}}=0.7 \\
M_{\mathrm{BH}}=10\end{array}$ & $\begin{array}{c}M_{\mathrm{WD}}=0.7 \\
M_{\mathrm{BH}}=3\end{array}$ & $\begin{array}{c}M_{\mathrm{WD}}=1.1 \\
M_{\mathrm{BH}}=10\end{array}$ & $\begin{array}{c}M_{\mathrm{WD}}=1.1 \\
M_{\mathrm{BH}}=3\end{array}$ \\
\hline$a \equiv j c / G M_{\mathrm{BH}}$ & 0.21 & 0.54 & 0.31 & 0.69 \\
$E_{\nu, \bar{\nu}}^{\max }\left(10^{49} \mathrm{Erg}\right)^{\mathrm{a}}$ & 0.001 & 0.003 & 3 & 50 \\
$E_{\mathrm{rot}}\left(10^{49} \mathrm{erg}\right)^{\mathrm{b}}$ & $\sim 0.1$ & $\sim 1$ & $\sim 1$ & $\sim 4$ \\
$M_{\text {axis }}\left(\mathrm{M}_{\odot}\right)^{\mathrm{c}}$ & $10^{-5}\left(<10^{-5}\right)$ & $10^{-4}\left(<10^{-5}\right)$ & $10^{-3}\left(<10^{-5}\right)$ & $10^{-3}\left(<10^{-5}\right)$ \\
$\left(E_{\nu, \bar{\nu}} / M_{\text {axis }} c^{2}\right)^{\mathrm{d}}$ & $>5 \times 10^{-4}$ & $>1.5 \times 10^{-3}$ & $>2.5$ & $>25$ \\
& & & & \\
\hline
\end{tabular}

${ }^{a} E_{\nu, \bar{\nu}}^{\max }$ is the neutrino/anti-neutrino annihilation energy released by setting the accretion rate equal to the mass-transfer rate. The luminosity is a function of both the accretion rate and the spin parameter $a$ (see Eq. 9) and we estimate the energy by integrating the luminosity assuming the black hole is not spinning before accretion sets in. The accretion rate can never exceed the mass-transfer rate, and hence, these energies are rough upper limits for the gamma-ray burst energy.

${ }^{b}$ We use the magnetic field estimate of Popham, Woosley, \& Fryer (1998) which assumes that the magnetic field is $10 \%$ of the equipartition energy, or $0.01 \rho v^{2}$.

${ }^{\mathrm{c}}$ This corresponds to the mass from the white dwarf which lies along the accretion disk axis and is likely to be swept up in the explosion for a beaming factor of 10(100).

${ }^{\mathrm{d}} E_{\nu, \bar{\nu}} / M_{\text {axis }} c^{2} \approx \gamma$ when $E_{\nu, \bar{\nu}} / M_{\text {axis }} c^{2} \gg 1$. We assume beaming factors of 100. 


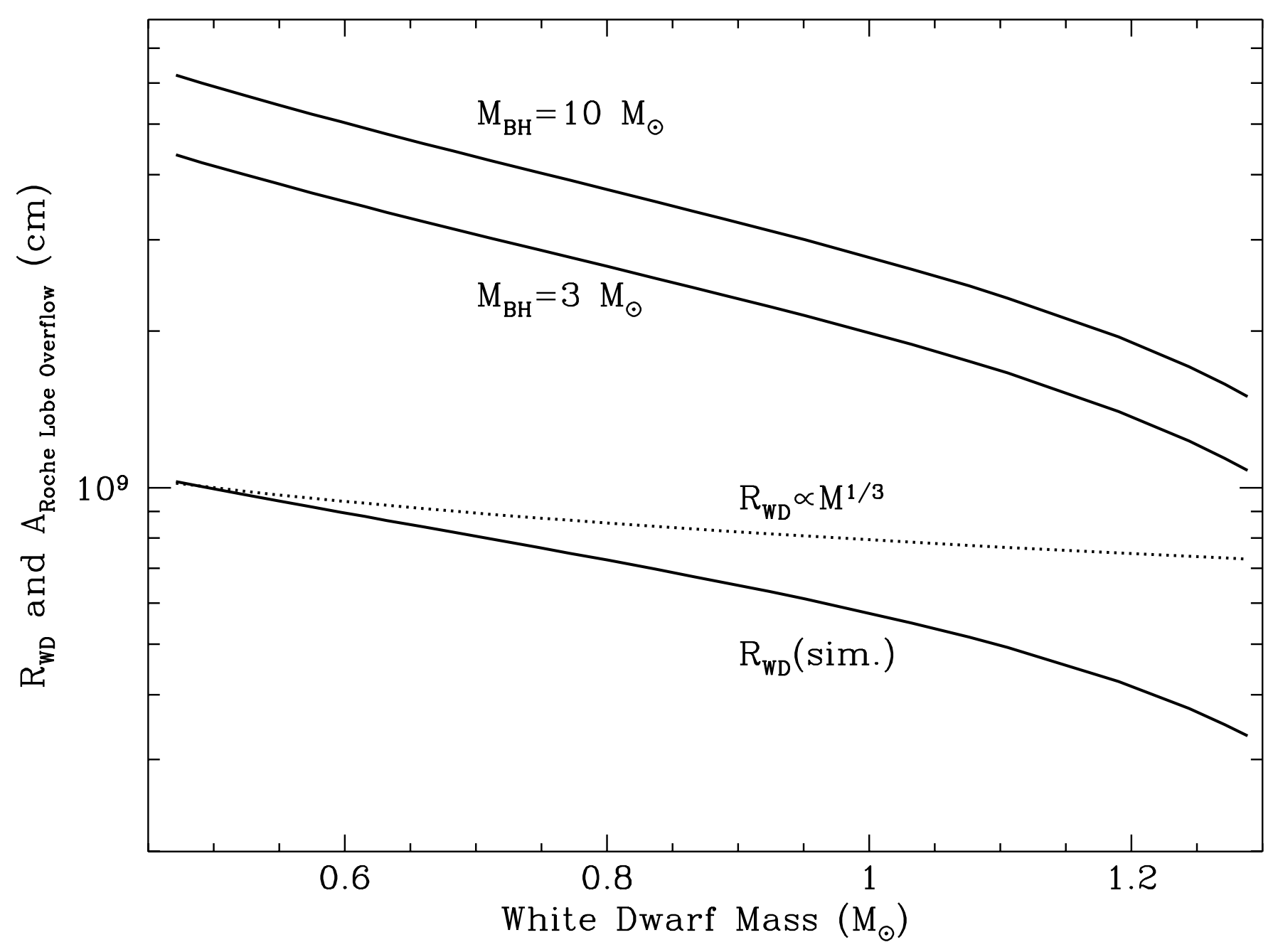

Fig. 1. - White dwarf radii and separations at which Roche-lobe overflow occurs vs. white dwarf mass. The dotted line shows the white dwarf radius using eq. (1) in comparison to our simulated radii. The orbital separations are given for two black hole masses $\left(3,10 \mathrm{M}_{\odot}\right)$ and demark the limit within which the white dwarf overfills its Roche lobe. 

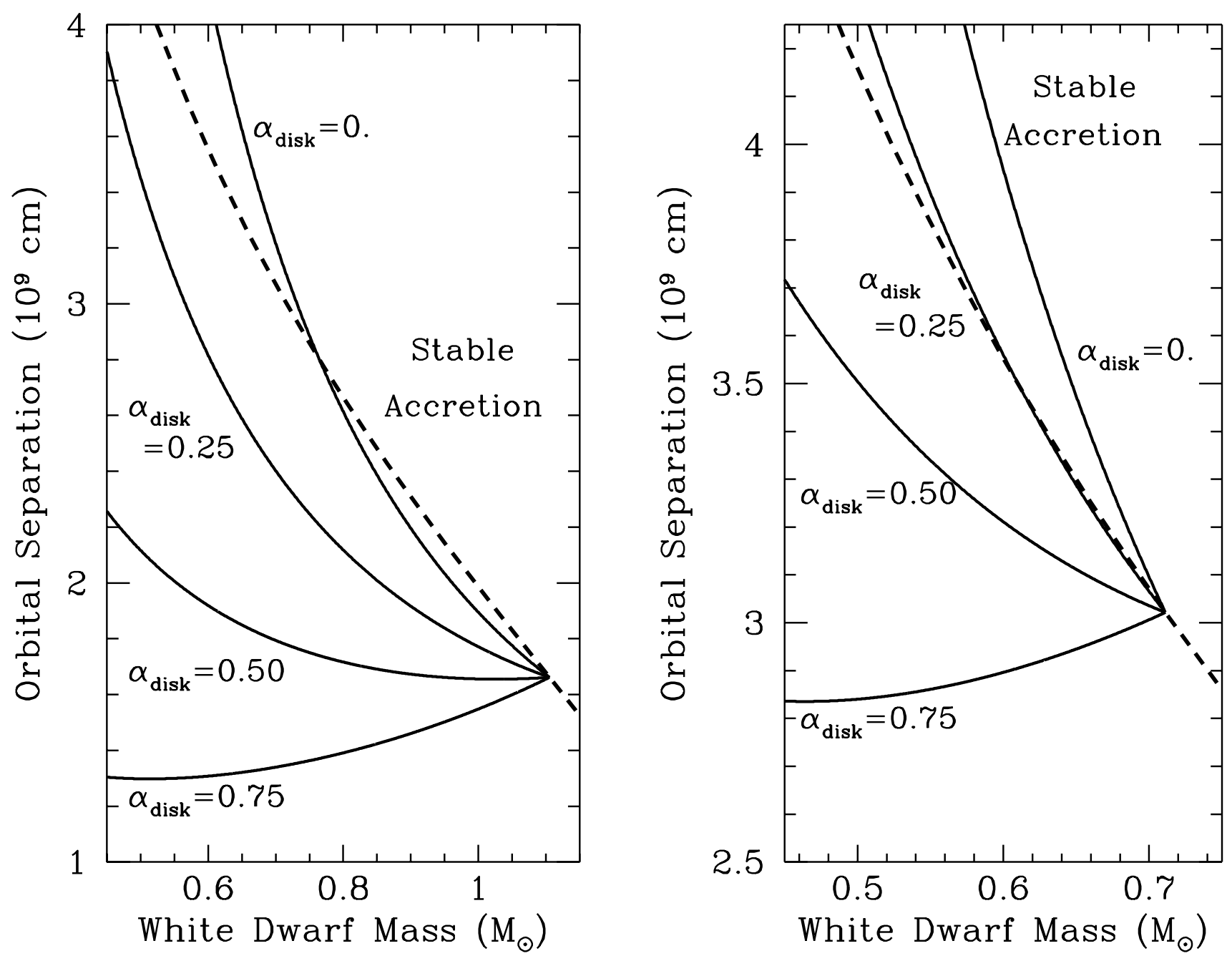

Fig. 2.- Evolutionary paths of the binary separation as the white dwarf accretes onto the black hole for a range of $j_{\text {disk }}$ values. The critical Roche-lobe separation is plotted for comparison. If the separation remains above this critical separation, stable accretion occurs. Otherwise, the accretion is unstable and the white dwarf quickly accretes onto the black hole. 

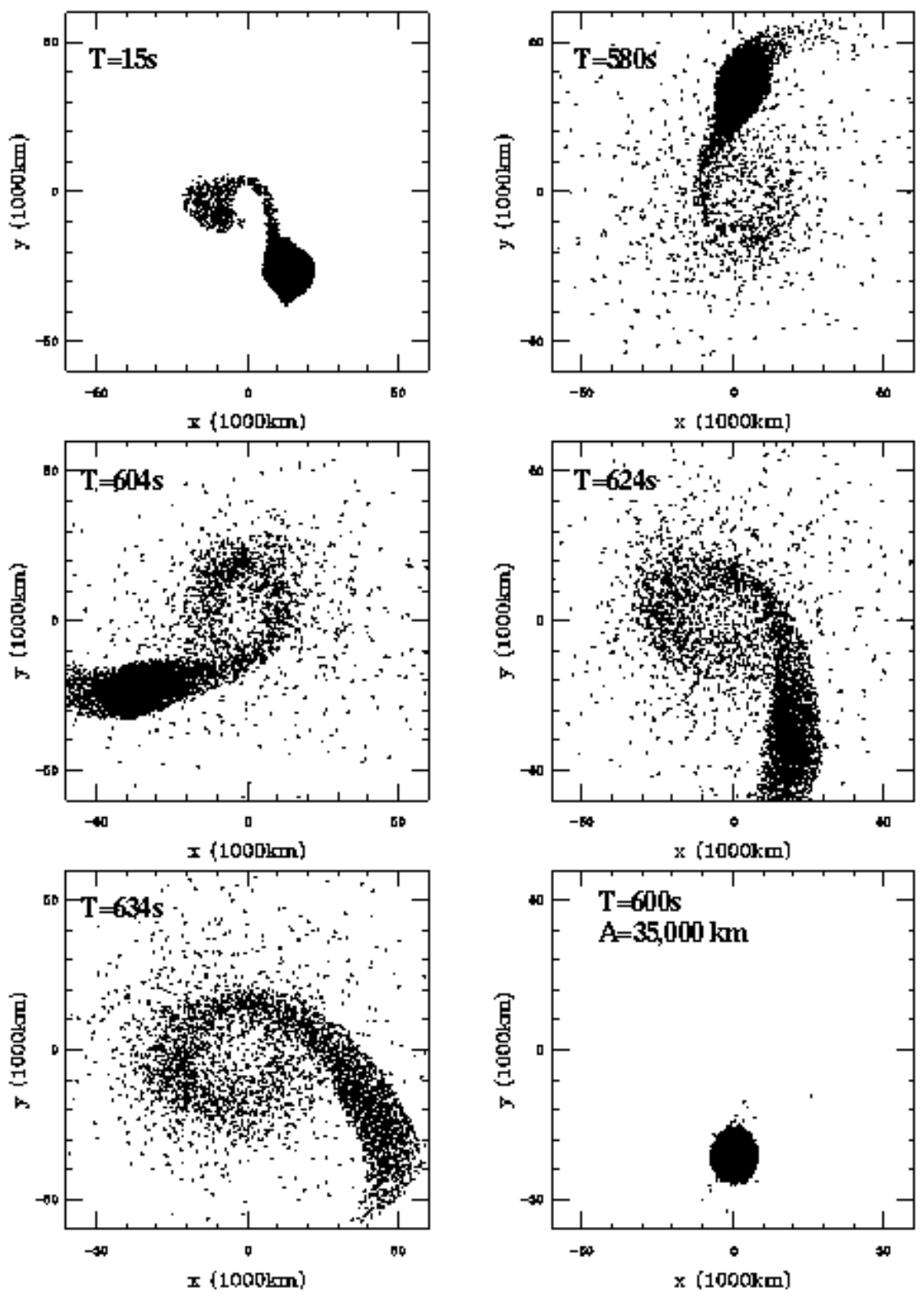

Fig. 3.- Time evolution of a $M_{\mathrm{WD}}=1.1 \mathrm{M}_{\odot}, M_{\mathrm{BH}}=3.0 \mathrm{M}_{\odot}$ simulation. Here we show slices about the z-axis from $-10000-10000 \mathrm{~km}$. Note that very little accretion occurs for over 600 s and then, very rapidly, the white dwarf is torn apart. However, for orbital separations just $20 \%$ further out (lower right panel), there is no mass transfer. This suggests our initial conditions are roughly accurate. 

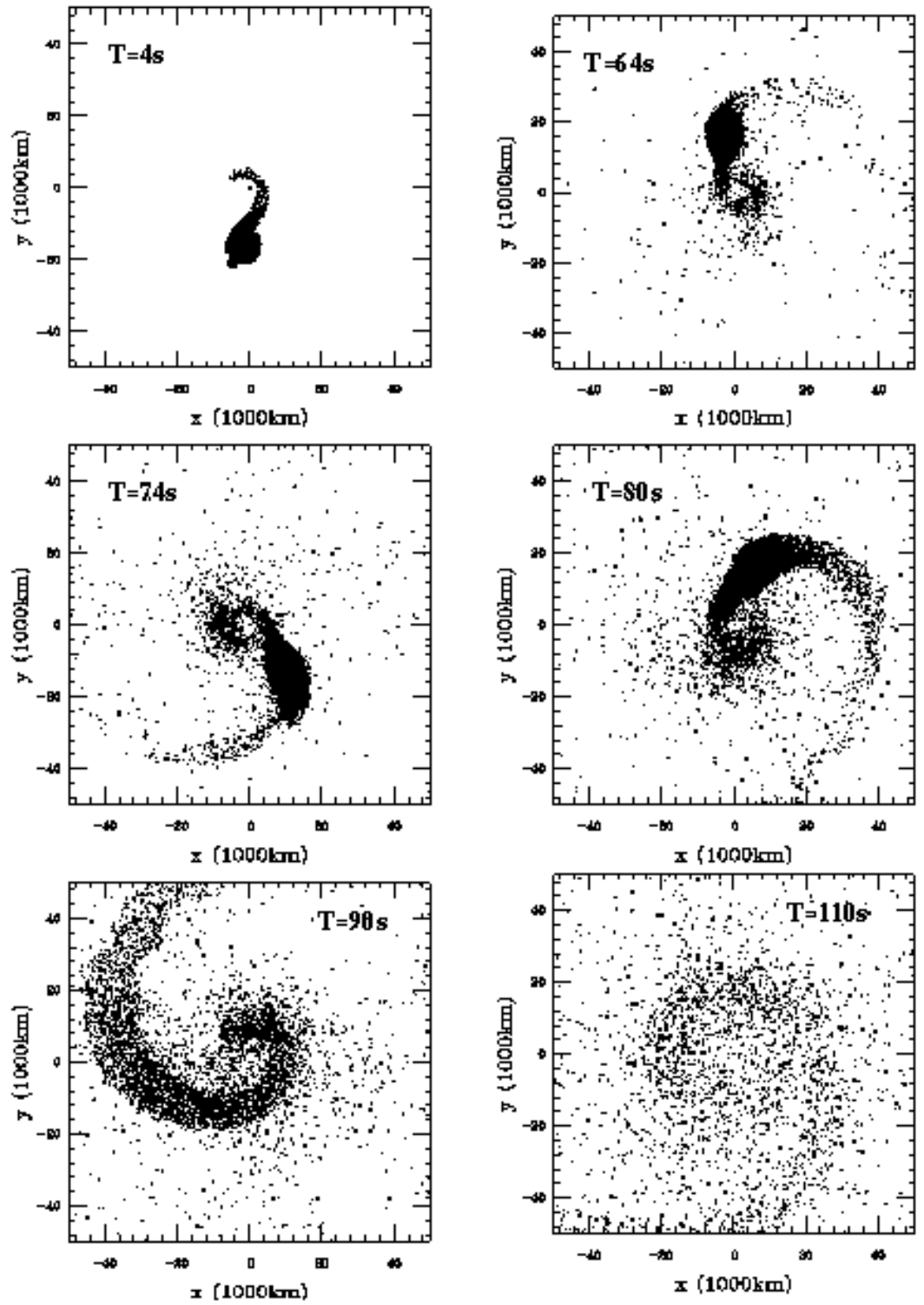

Fig. 4.- Time evolution of a $M_{\mathrm{WD}}=1.1 \mathrm{M}_{\odot}, M_{\mathrm{BH}}=3.0 \mathrm{M}_{\odot}$ simulation. Here we show slices about the z-axis from $-10000-10000 \mathrm{~km}$. Note that very little accretion occurs for over $70 \mathrm{~s}$ and then, very rapidly, the white dwarf is torn apart. In the last slide $(T=110 \mathrm{~s})$, nearly all traces of the white dwarf have been removed and half of the white dwarf mass has been accreted onto the black hole. 


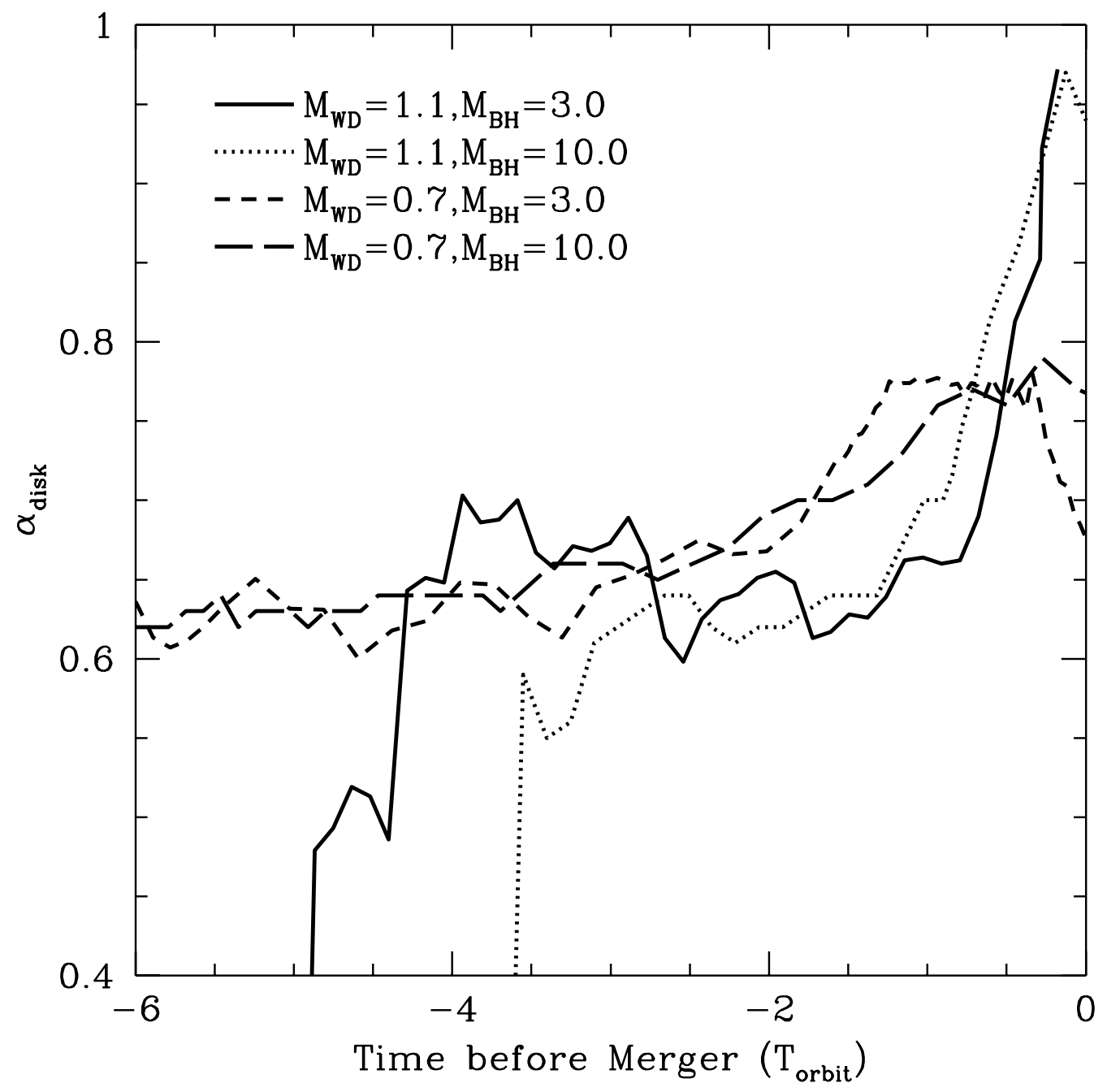

Fig. 5.- The specific angular momentum $\left(j_{\text {disk }}\right)$ of the disk (in units of the white dwarf specific angular momentum) as a function of time (in orbital time). The orbital times of the systems are given in Table 1. As the white dwarf is disrupted, its entire angular momentum is put in the disk. 

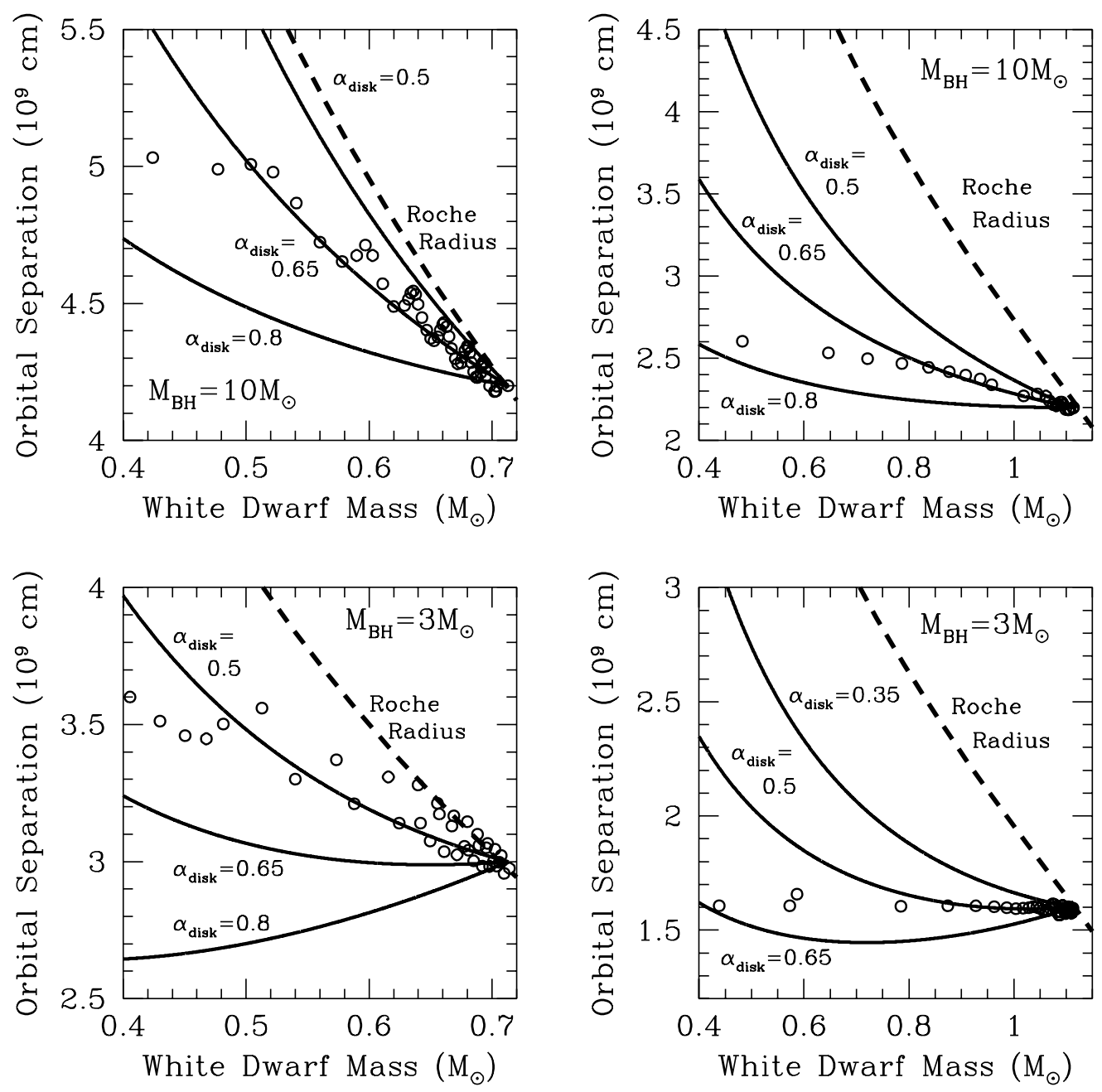

Fig. 6.- Orbital separation as a function of white dwarf mass. The circles are the data from the simulations and the solid lines denote the predicted separations from equation (3). The dashed line is the separation at which the white dwarf overfills its Roche radius. Note that after losing $\sim 0.2 \mathrm{M}_{\odot}$, the orbital separation evolution remains constant. This occurs as the white dwarf is torn apart by tidal forces. 


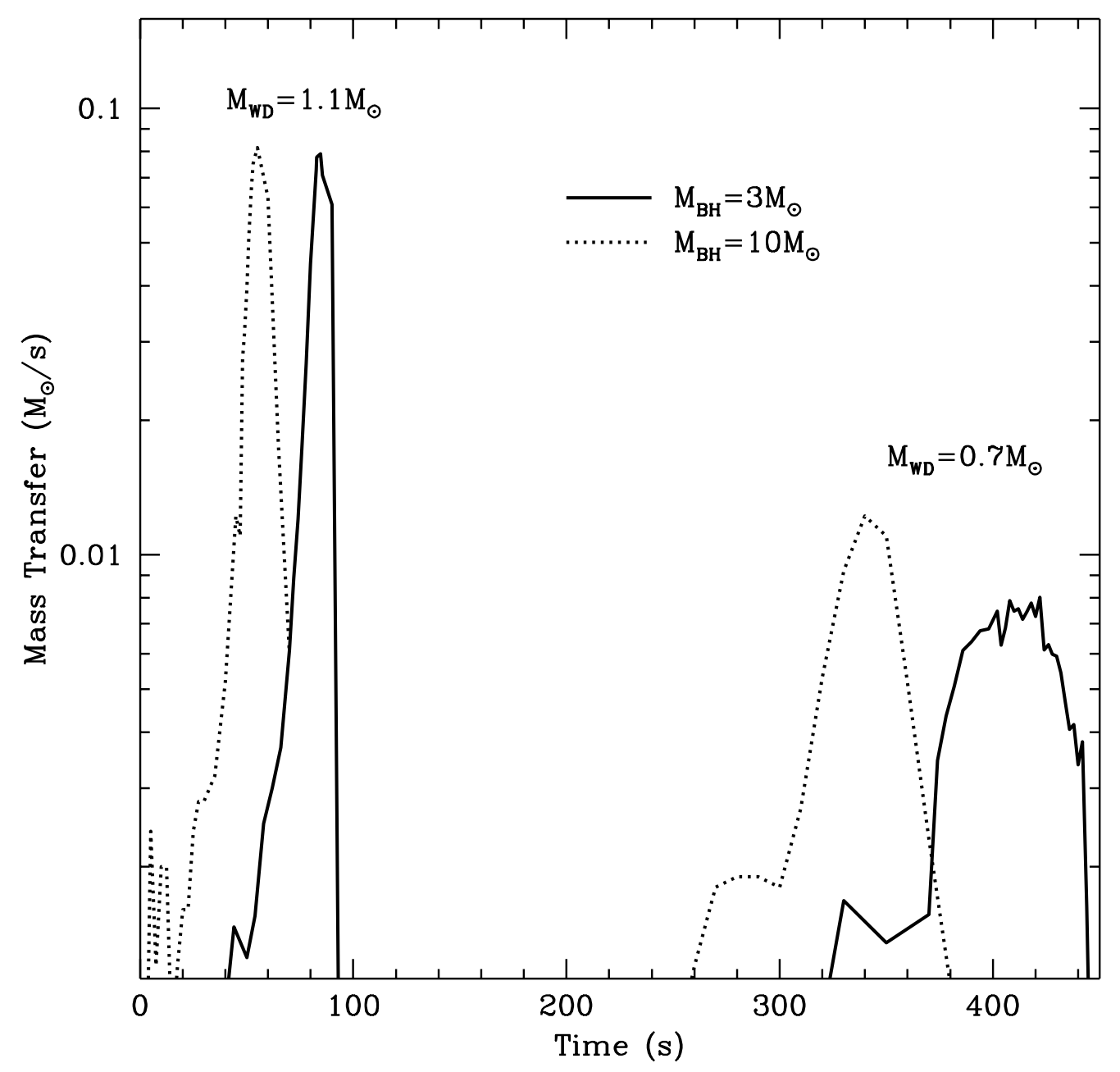

Fig. 7.- Mass Transfer Rate vs. time for the 4 white dwarf/black hole mergers. The more massive white dwarfs merge more quickly and have correspondingly higher mass-transfer rates. This rate gives a maximum for the disk accretion rate onto the black hole. 


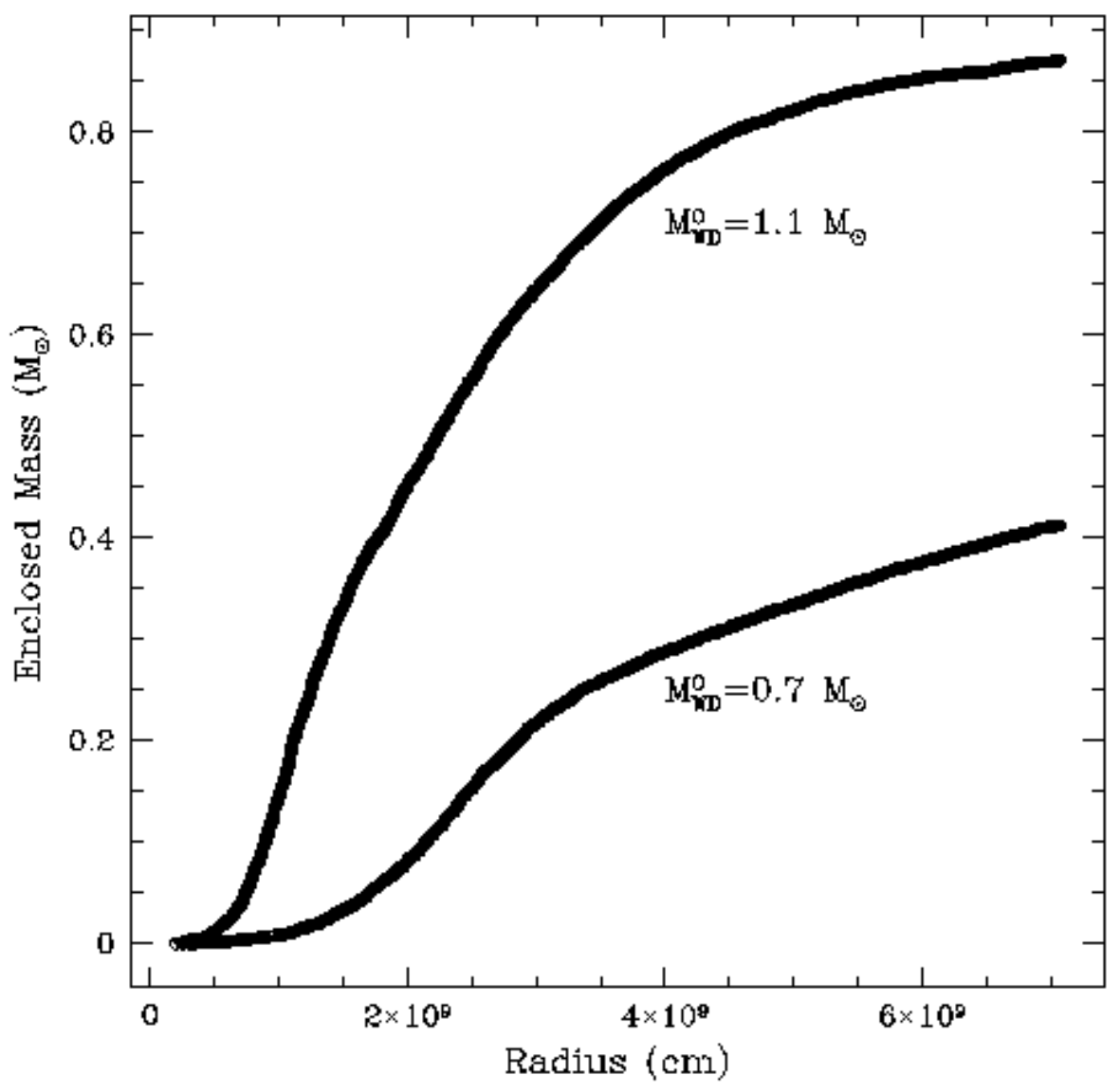

Fig. 8. - Enclosed Mass vs. radius just after white dwarf disruption. The accretion rate of this material onto the disk is roughly given by: $\dot{M}_{\text {acc }} \approx \alpha \Omega M_{\text {disk }}$. 

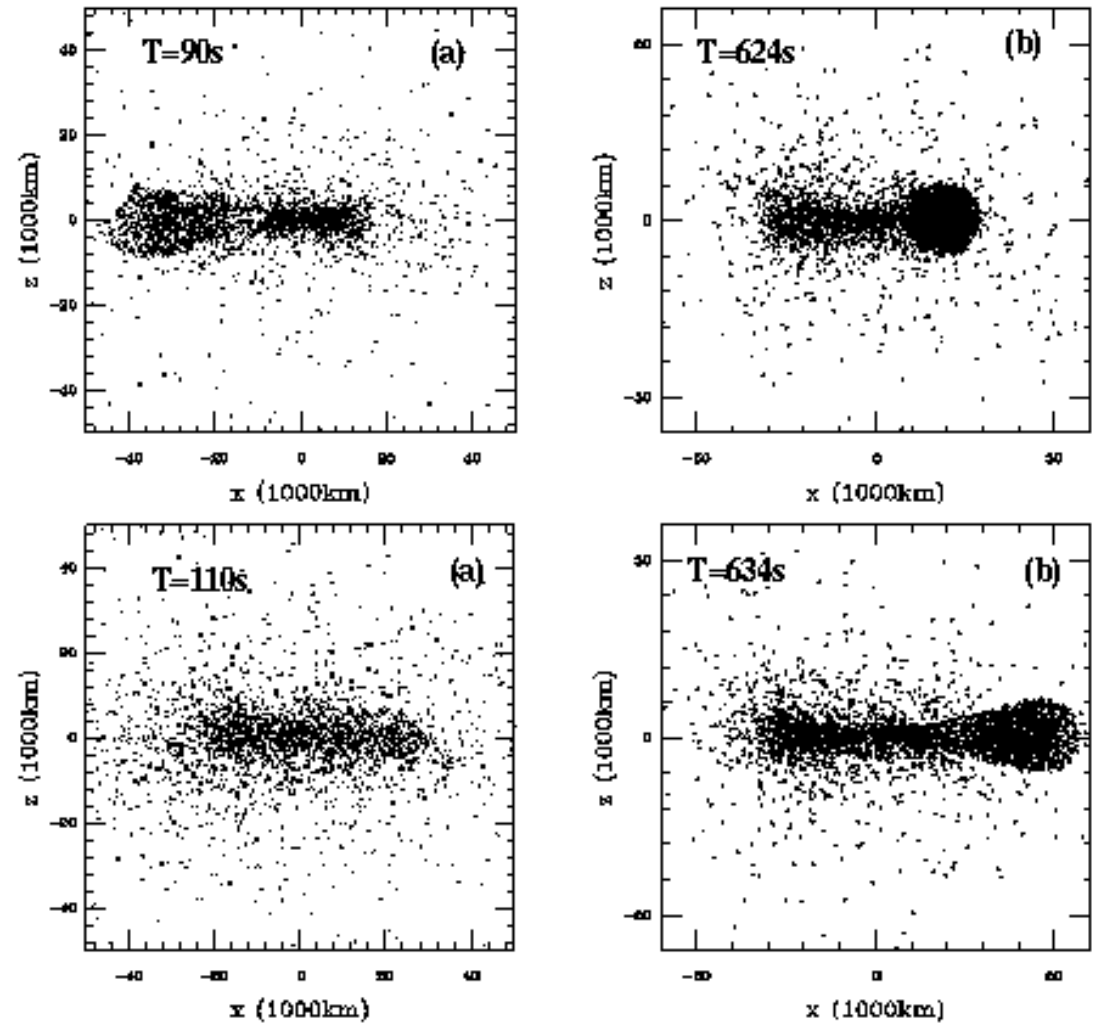

Fig. 9.- Distribution of the white dwarf material along the rotation axis for our two $M_{\mathrm{BH}}=3 \mathrm{M}_{\odot}$ simulations. Any GRB explosion must plow through this material (and possibly sweep it up) as it expands. If the explosion is beamed, the total swept up mass is very small (see Table 2) and will not effect the GRB. 